\title{
A correlation between capsid protein VP2 and the plaque morphology of African horse sickness virus in cell culture
}

\begin{abstract}
:
The attenuated live virus vaccine that is used in South Africa to protect against African horse sickness infection, was developed more than 50 years ago. With the selection of the vaccine strains by cell culture passage, a correlation between the size of plaques formed in monolayer Vero cultures and attenuation of virus virulence in horses was found. The large plaque phenotype was used as an indication of cell culture adaptation and strongly correlated with attenuation of virulence in horses. There was never any investigation into the genetic causes of either the variation in plaque size, or the loss of virulence. An understanding of the underlying mechanisms of attenuation would benefit the production of a safer AHSV vaccine. To this end, the genomes of different strains of two African horse sickness isolates, producing varying plaque sizes, were compared and the differences between them identified. This comparison suggested that proteins VP2, VP3, VP5 and NS3 were most likely involved in the determination of the plaque phenotype. Comparison between genome sequences (obtained from GenBank) of low and high passage strains from two additional serotypes, indicated that VP2 was the only protein with amino acid substitutions in all four serotypes. The amino acid substitutions all occurred within the same hydrophilic area, resulting in increased hydrophilicity of VP2 in the large plaque strains.
\end{abstract}

\section{List of authors:}

Mathilde L. Schade-Weskott (ORCID iD: 0000-0003-4130-6543)

Agricultural Research Council - Onderstepoort Veterinary Institute, 100 Old Soutpan Rd, Onderstepoort, South Africa; Department of Veterinary Tropical Diseases, Faculty of Veterinary Science, University of Pretoria)

Antoinette van Schalkwyk

Agricultural Research Council - Onderstepoort Veterinary Institute, 100 Old Soutpan Rd, Onderstepoort, South Africa

\section{J. J. O. Koekemoer}

Agricultural Research Council - Onderstepoort Veterinary Institute, 100 Old Soutpan Rd, Onderstepoort, South Africa

\section{Keywords}

African horse sickness virus; attenuation; plaque phenotype; VP2-gene 


\section{Introduction}

African horse sickness virus (AHSV) is an orbivirus of the family Reoviridae [1]. The AHSV genome consists of ten double-stranded RNA (dsRNA) segments, which are enveloped in two concentric protein layers [2-5]. The AHSV genome codes for seven structural virus proteins (VP1-7) and four nonstructural proteins (NS1-4) [6-8]. The outer capsid consists of VP2 and VP5, where the former is responsible for the determination of the virus serotype as well as virus entry into the host cell $[9,10]$, while the latter has a membrane permeabilizing activity [11-13]. The major proteins of the virus core are VP3 and VP7, with VP3 forming a scaffold around the dsRNA genome and enclosing the minor proteins VP1, VP4 and VP6 [14]. The minor proteins form a transcriptional complex, where VP1 functions as a RNA-dependent RNA polymerase [15-18], VP4 stabilizes transcripts through capping and methylation [19-21], and VP6 is an ATP-dependent helicase which unwinds the dsRNA genome prior to transcription [22-25]. The non-structural proteins are not part of the virus capsid, but are produced during the infection cycle. NS1 plays a role in the upregulation of viral gene translation as well regulation of virus release [26,27], NS2 is responsible for the formation of virus inclusion bodies (VIB) in the cell cytosol [28], NS3 is involved in viral egress [29-31] and NS4 binds dsDNA and is an interferon antagonist, thus inhibiting the host immune reaction $[7,8,32]$

AHSV infection causes African horse sickness (AHS) which is an economically important disease that affects all equids [33], with particularly serious clinical consequences in immunologically naïve horses. It is estimated to have a mortality rate of $90-95 \%$ in unprotected horses. The virus is transmitted during blood-feeding by several Culicoides sp. midges [34-37] and is endemic to most sub-Saharan countries [38-41] where live attenuated viruses are used as a polyvalent vaccine [42].

During the development of the cell culture adapted, live attenuated viruses, it was observed that a correlation exists between plaque phenotype and the attenuation of the virus virulence in horses. Strains with a small plaque phenotype in Vero monolayers produced severe clinical signs in horses, while large plaque strains caused a mild febrile reaction, if any [42,43]. The large plaque phenotype is rarely observed when infecting cell cultures with low passage field isolates, and selected isolates were thus serially passaged on BHK-21 before stable large plaque variants were selected. Candidate vaccine viruses selected based on large plaque morphology, were non-pathogenic in horses. It was thus concluded that plaque size can be used as a marker for cell culture adaptation and attenuation [42].

In this study, the complete genomes of two AHSV field isolates and their cell culture-adapted passages were sequenced and compared to identify the potential genetic causes of the change in plaque morphology and compared to the known sequences of virulent and attenuated strains of two more AHSV isolates [44-46].

\section{Materials and Methods}

Mammalian cell culture and AHSV strains

Baby hamster kidney (BHK-21;) and BSR cell monolayers [47,48] were cultured in $75 \mathrm{~cm}^{2}$ flasks using BioWhittaker Dulbecco's Modified Eagle's Medium (DMEM) (Lonza), supplemented with 5\% (v/v) foetal 
bovine serum (FBS) (HyClone), 1\% (v/v) L-glutamine [200 mM in a $0.85 \%(\mathrm{w} / \mathrm{v}) \mathrm{NaCl}$ solution, Lonza] and $1 \%(\mathrm{v} / \mathrm{v})$ PSA (10 $000 \mathrm{U} / \mathrm{ml}$ of penicillin, $10000 \mu \mathrm{g} / \mathrm{ml}$ of streptomycin and $25 \mu \mathrm{g} / \mathrm{ml}$ of amphotericin $\mathrm{B}$; HyClone). The flasks were incubated at $37^{\circ} \mathrm{C}$, with $5 \% \mathrm{CO}_{2}$, in a humidified incubator.

Cell culture inoculations were prepared by resuspending lyophilized preparations of different strains (Table 1) in Hanks balanced salt solution (Lonza) and infecting BHK-21 monolayers at a confluency of $70-80 \%$ with the virus. Monolayers were incubated for 3-4 days, or until cytopathic effects were observed. Infected cell monolayers were used for infections and RNA extraction.

\section{Plaque assays}

Plaque assays were performed using a modified protocol of Oellerman (1970) [49]. BSR monolayers, at a confluency of $90 \%$ in 6 -well cell culture plates, were infected with ten-fold serial dilutions of each virus. The inoculum was removed $2 \mathrm{~h}$ post infection and an agarose overlay [2\% (v/v) FBS and $0.8 \%$ (v/v) agarose (SeaPlaque $\AA$ agarose, Lonza) in Eagle's Minimum Medium (Gibco)] applied. Plates were incubated for six days before staining with MTT [(3-(4, 5-dimethylthiazol-2-yl)-2, 5-diphenyltetrazolium bromide] to a final concentration of $0.25 \mathrm{mg} / \mathrm{ml}$. Cultures were then incubated overnight and the plaques visualized. Plaques were photographed, and their sizes determined with ImageJ $1.49 \mathrm{v}$ software [50] using the plate well diameter $(33.9 \mathrm{~mm})$ as reference. The diameters of 100 plaques were determined for each of the virus strains listed in Table 1.

Table 1 List of viruses propagated in this study

\begin{tabular}{|c|c|c|c|c|}
\hline $\begin{array}{l}\text { Virus } \\
\text { name }\end{array}$ & In text reference & Isolate no.* & Serotype & Passage history \\
\hline $29 / 623 S$ & AHSV-1 3S & $29 / 62$ & 1 & 3 suckling mouse brain \\
\hline 29/62 Vero & AHSV-1 Vero & $29 / 62$ & 1 & 3 suckling mouse brain, 1 Vero $(22 / 05 / 98)$ \\
\hline 29/62 SJ & AHSV-1 SJ & $29 / 62$ & 1 & 3 suckling mouse brain, 1 Vero $(09 / 01 / 98)$ \\
\hline 29/62 MP & AHSV-1 MP & $29 / 62$ & 1 & $\begin{array}{l}3 \text { mouse brain, } 10 \text { BHK-21, } 4 \text { medium plaque picks, } \\
3 \text { Vero, } 1 \text { BHK-21 }\end{array}$ \\
\hline $\begin{array}{l}29 / 62 \\
L P(A)\end{array}$ & AHSV-1 LP(A) & $29 / 62$ & 1 & $\begin{array}{l}3 \text { suckling mouse brain, } 10 \text { BHK-21, } 3 \text { large plaque } \\
\text { picks }(A), 4 \text { Vero }\end{array}$ \\
\hline $\begin{array}{l}29 / 62 \\
L P(B)\end{array}$ & AHSV-1 LP(B) & $29 / 62$ & 1 & $\begin{array}{l}3 \text { suckling mouse brain, } 10 \text { BHK-21, } 3 \text { large plaque } \\
\text { picks (B), } 4 \text { Vero }\end{array}$ \\
\hline $82 / 613 S$ & AHSV-2 3S & $82 / 61$ & 2 & 3 suckling mouse brain \\
\hline 82/61 LP & AHSV-2 LP & $82 / 61$ & 2 & $\begin{array}{l}3 \text { suckling mouse brain, } 10 \text { BHK-21, } 3 \text { large plaque } \\
\text { picks, } 5 \text { Vero }\end{array}$ \\
\hline
\end{tabular}




\section{Statistical analysis}

Plaque diameter data were used in two-sample comparisons to determine whether there was a statistically significant difference between the plaque sizes produced by the AHSV strains. The MannWhitney $U$ test [51], was performed using a two-tailed hypothesis and a significance level of 0.05 .

Preparation and sequencing of AHSV genomic cDNA

Double-stranded RNA was isolated from infected BHK-21 cell culture monolayers and cDNA prepared and amplified as previously described [52]. The amplicons were sequenced using the MiSeq (Illumina) next generation sequencing (NGS) platform.

Genome assembly and analysis

The sequence data were analysed in CLC Genomics Workbench version 8.5 (Qiagen; https://www.qiagenbioinformatics.com/). Adaptor sequences were removed and the trim reads de novo assembled as well as mapped to the reference AHSV-1 29/62 1A1V (unpublished) or AHSV-2 82/61 3S [46]. A consensus sequence was generated from contigs constructed through both de novo assembly and reference mapping. The data were used to determine the genetic composition within the virus populations by detecting variations at a frequency higher than $5 \%$ and with a quality score of more than 30. The complete genome sequences of all the viruses determined in this study were submitted GenBank [53] under accession numbers KY471465 - KY471544. Both nucleotide and amino acid sequences between the strains of a single isolate were compared.

\section{Comparison of AHSV sequences from other serotypes}

Full genome sequences of serotype 4 (AHSV-4 32/62 1S) and serotype 6 (AHSV-6 02/75 4S), as well as their respective live attenuated viruses were obtained from Genbank (AHSV-Labstr/ZAF/1998/OBP116 serotype 4 [Vac4] and AHSV-6/Labstr/ZAF /1998/OBP-252.1 [Vac6]) [44-46]. Differences between the nucleotide and amino acid sequences between the strains of each serotype were determined and compared to those previously determined for AHSV-1 29/62 and AHSV-2 82/61.

\section{Hydropathy and antigenicity plots}

The amino acid sequence of VP2 protein from each virus was submitted to the ExPASy server [54] to predict both the hydropathy and the antigenicity of the proteins. Hydropathy was predicted using KyteDoolittle algorithm [55], while antigenicity was predicted using the method of Welling et al. [56]. In both cases a window size of 9 was used.

\section{Results}

Plaque sizes fall into three categories

To correlate genome sequences to a phenotype, the diameters of $100 \mathrm{pfu}$ from each virus were determined (Table 1). These were compared between the viruses with different passage history within 
a serotype, to determine whether plaques could be categorized as small or large. The average plaque size for each of the virus strains have been summarised in Table 2.

Table 2 Average plaque size of the AHSV-1 and AHSV-2 strains

\begin{tabular}{|l|l|l|l|l|l|l|l|l|}
\hline & $\begin{array}{l}\text { AHSV-1 } \\
\text { 29/62 3S }\end{array}$ & $\begin{array}{l}\text { AHSV-1 } \\
\text { 29/62 SJ }\end{array}$ & $\begin{array}{l}\text { AHSV-1 } \\
\text { 29/62 Vero }\end{array}$ & $\begin{array}{l}\text { AHSV-1 } \\
\text { 29/62 MP }\end{array}$ & $\begin{array}{l}\text { AHSV-1 } \\
\text { 29/62 } \\
\text { LP(A) }\end{array}$ & $\begin{array}{l}\text { AHSV-1 } \\
\text { 29/62 } \\
\text { LP(B) }\end{array}$ & $\begin{array}{l}\text { AHSV-2 } \\
82 / 613 S\end{array}$ & $\begin{array}{l}\text { AHSV-2 } \\
82 / 61 \text { LP }\end{array}$ \\
\hline $\begin{array}{l}\text { Avg. } \\
\text { diameter } \\
(\mathrm{mm})\end{array}$ & 0.417 & 0.615 & 0.606 & 0.681 & 2.285 & 1.955 & 0.389 & 1.462 \\
\hline $\begin{array}{l}\text { Size } \\
\text { distribution } \\
\text { (mm) }\end{array}$ & $\begin{array}{l}0.227- \\
0.721\end{array}$ & $\begin{array}{l}0.42- \\
1.411\end{array}$ & $0.421-0.995$ & $\begin{array}{l}0.488- \\
1.186\end{array}$ & $\begin{array}{l}1.098- \\
3.212\end{array}$ & $\begin{array}{l}0.98- \\
3.036\end{array}$ & $\begin{array}{l}0.274- \\
0.524\end{array}$ & $\begin{array}{l}2.752- \\
2.59\end{array}$ \\
\hline $\begin{array}{l}\text { Standard } \\
\text { deviation }\end{array}$ & 0.069 & 0.128 & 0.1 & 0.115 & 0.458 & 0.489 & 0.049 & 0.421 \\
\hline
\end{tabular}

AHSV-1 29/62 strains (AHSV-1; Fig. 1) fell into small (avg. diameter below $0.5 \mathrm{~mm}$ ), intermediate (avg. diameter between 0.5 and $1 \mathrm{~mm}$ ) and large (avg. diameter above $1 \mathrm{~mm}$ ) plaque phenotypes. These size categories were determined to be statistically significantly different from each other. Viruses in the large plaque category $[A H S V-1 \mathrm{LP}(\mathrm{A})$ and $\mathrm{LP}(\mathrm{B})]$ did not differ from each other, however both are statistically different from the viruses in the intermediate (AHSV-1 MP, SJ and Vero) and small (AHSV$13 S$ ) plaque categories, with a $p$-value of less than 0.05 . Similarly, the medium plaque viruses did not differ from each other, but there was a statistically significant difference between these viruses and the small plaque virus $(p$-value $<0.05)$

The results of the plaque size analysis of strains AHSV-2 $3 S$ and LP indicated that the former had a small plaque phenotype, while the latter had a large plaque phenotype (AHSV-2; Fig. 1). The MannWhitney $U$ test indicated that these two populations were significantly different from each other ( $p$-value $<0.05)$.

\section{Sequence analysis}

There were 47 single nucleotide polymorphisms (SNPs) detected between the six AHSV-1 29/62 strains, while 15 SNPs were detected between the two strains of AHSV-2 82/61. Comparisons between the genome sequences of reference strain and live attenuated viruses from serotype 4 and 6 had 27 and 46 SNPs respectively.

The amino acid sequences inferred from the genome sequences were also compared to determine the effect of each SNPs. The AHSV-1 strains had 13 amino acid substitutions between them, none of which occurred in proteins VP6, VP7, NS2 or NS4 (Fig. 2a). There were 10 amino acid residue substitutions between AHSV-2 $3 S$ and LP, with no substitutions occurring in VP1, VP4, VP7 and NS1 (Fig. 2b). All 
Fig. 1 Plaque diameters of the AHSV-1 and AHSV-2 virus strains. Average plaque size for each strain has been indicated with a stippled line

\section{Analysis of AHSV strain plaque sizes}

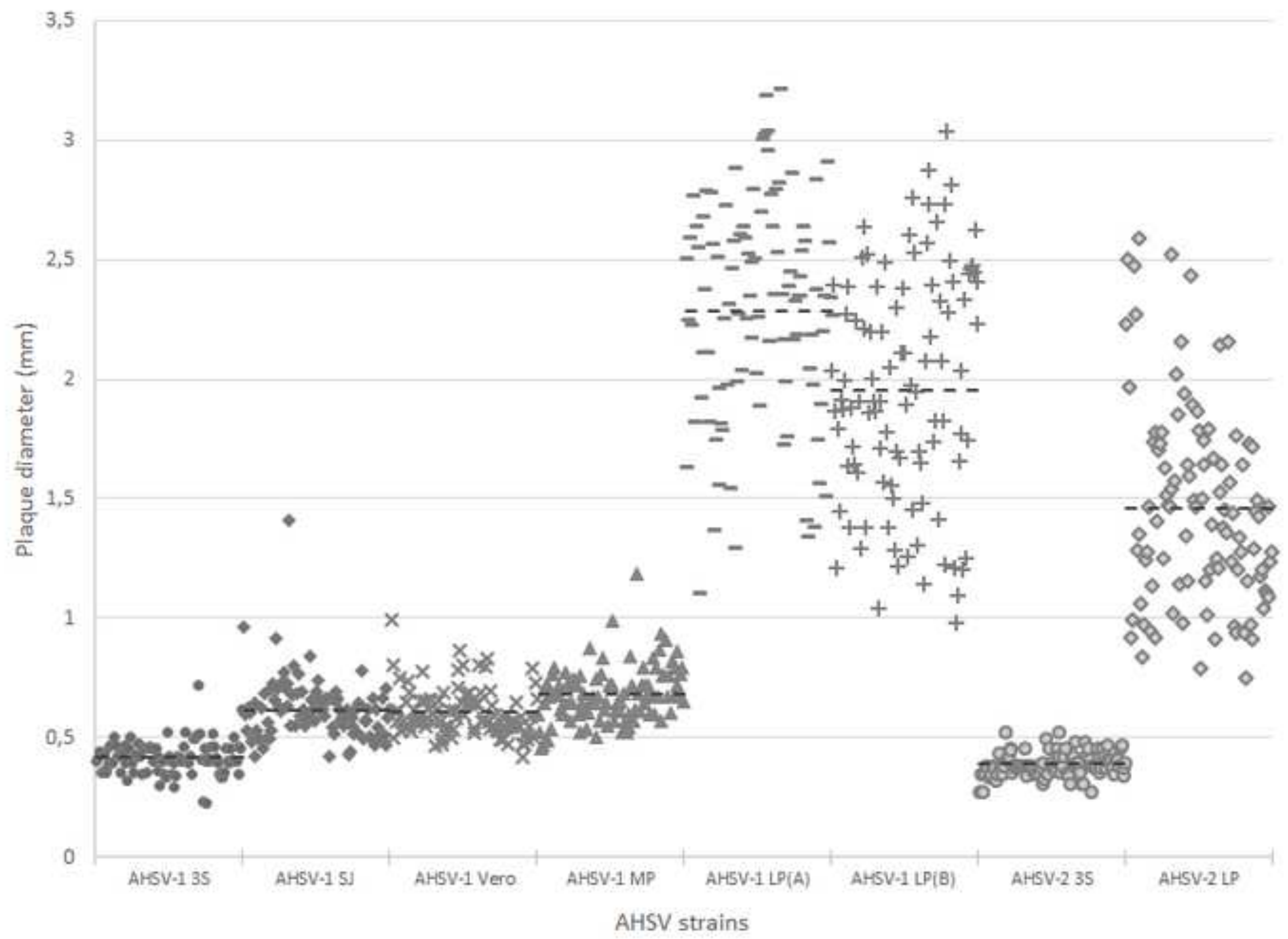


Fig. 2 Amino acid differences between the strains of a AHSV-1, b AHSV-2, c AHSV-4 and d AHSV-6. The approximate location of each amino acid substitution has been indicated by a black bar, and the type of change annotated

a

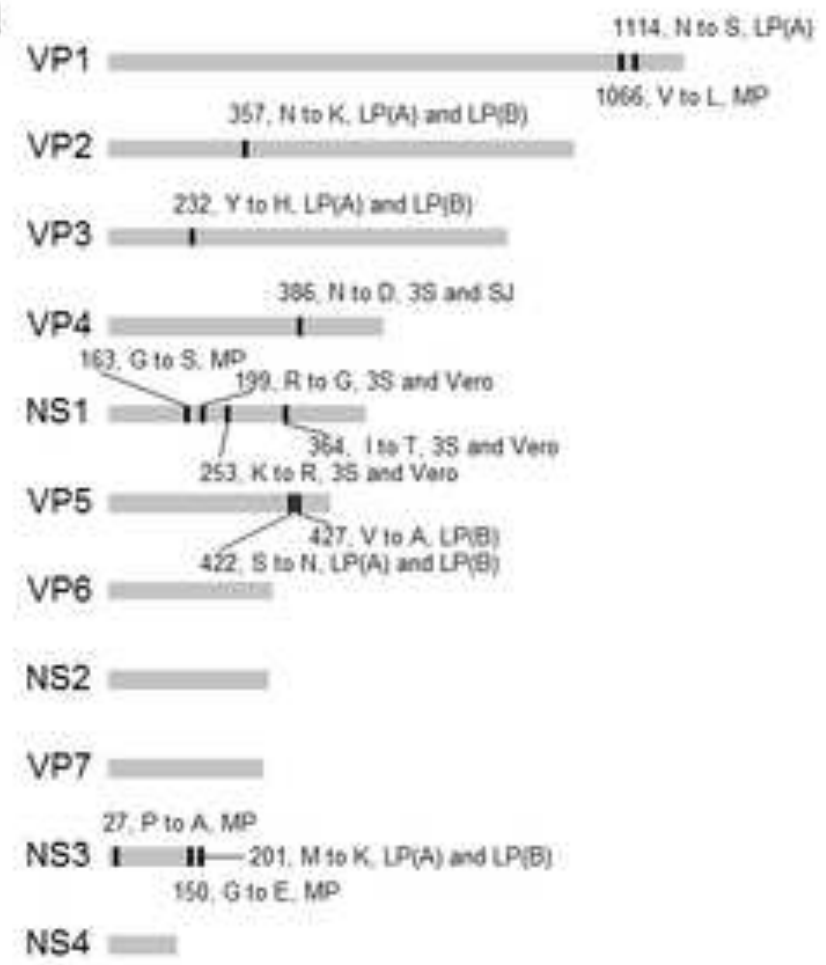

C

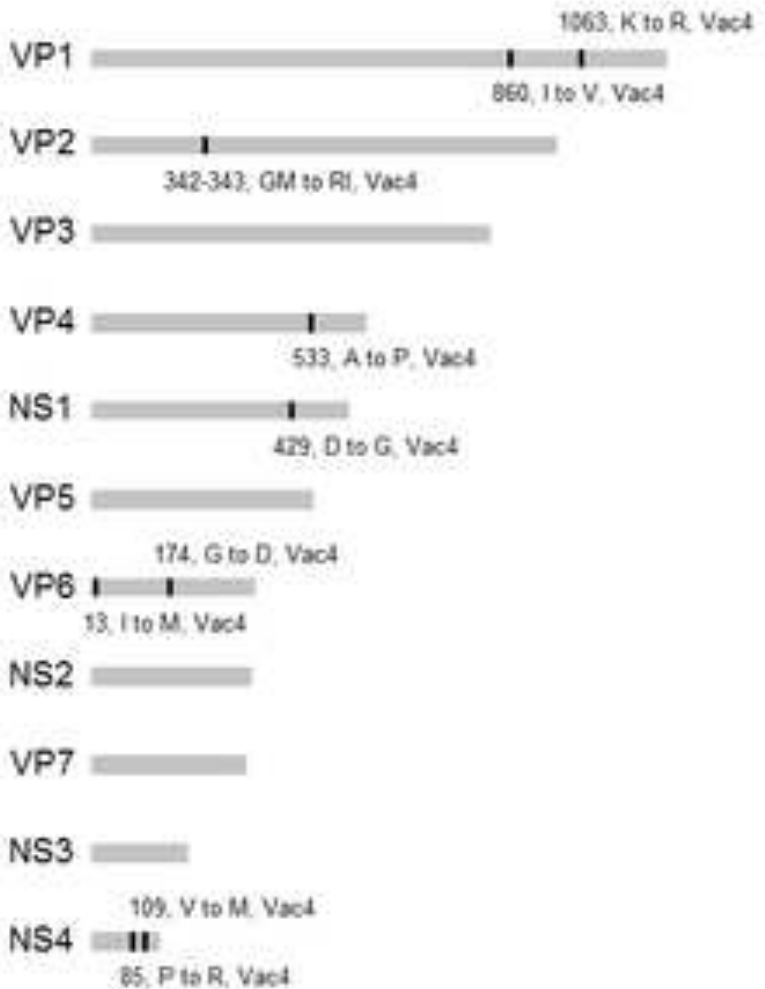

b

VP1
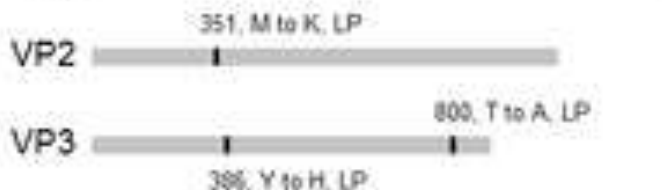

VP4

NS

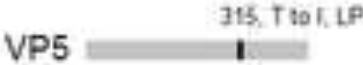

VP6 187. T to ti ip

153. 8 IDF. UP

NS2

19. L to S, LP.

VP7

NS3 296. Y Y 10 LP

NS4 11 $129: 010$ \$100. LP

95. P to 5, LP

d

VP1

1115. Ero to E, Vac6

141, W to V Vack

VP2

353. T\$S R. Vac6

VP3

VP4

NS1

VP5

VP6 $=1$

232, $w$ to $\mathrm{K}: \operatorname{Vac} 6$

NS2 $=1$

305. N100, Vace

VP7

NS3 $\prod_{147, \text { Nto D. Vaco }}^{208, L \text {, to } 5, \text { Vack }}$

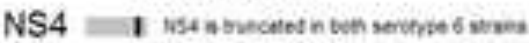

112 GE to E Vas6 
Fig. 3 Amino acid alignments showing substitutions between the strains of AHSV-1 and AHSV-2 in a VP2 and b NS3. The location of the amino acid substitutions between strains of each isolate is indicated with either a black (AHSV-1), red (AHSV-2), blue (AHSV-4) or green (AHSV-6) asterisks.

\section{a}

AHSV-1 29/62 LP(A) NS3 AHSV-1 29/62 LP(B) NS3 AHSV-1 29/62 MP NS3 AHSV-1 29/62 SJ NS3 AHSV-1 29/62 Vero NS3 AHSV-1 29/62 3S NS3 AHSV-2 82/61 LP NS3 AHSV-2 82/61 3S NS3 AHSV 4 Vac4 NS3 AHSV-4 32/62 1S NS3 AHSV-6 Vac6 NS3 AHSV-6 02/75 4SNS3

I
VSA ATKSWDGDSV
VS
VSA ATKSWDGDSV
VSA ATMSWDGDSV
VSA ATMSWDGDSV
VSA ATMSWDGDSV
VSA ATMSWDGDSV
VSA ATMSWDGDSV
VSA ATMSWDGDSV
VSA ASMSWSGDTE
VSA ASMSWSGDTE
VSA ASMSWNGDTE
VSA ASMSWNGDTE
VSA ASMSWN

b

AHSV-1 29/62 LP(A) VP2 AHSV-1 29/62 LP(B) VP2 AHSV-1 29/62 MP VP2 AHSV-1 29/62 SJ VP2 AHSV-1 29/62 Vero VP2 AHSV-1 29/62 3S VP2 AHSV-2 82/61 LP VP2 AHSV-2 82/62 3S VP2 AHSV-4 Vac4 VP2 AHSV-4 32/62 1S VP2 AHSV-6 Vac6 VP2 AHSV- 6 02/75 4S VP2

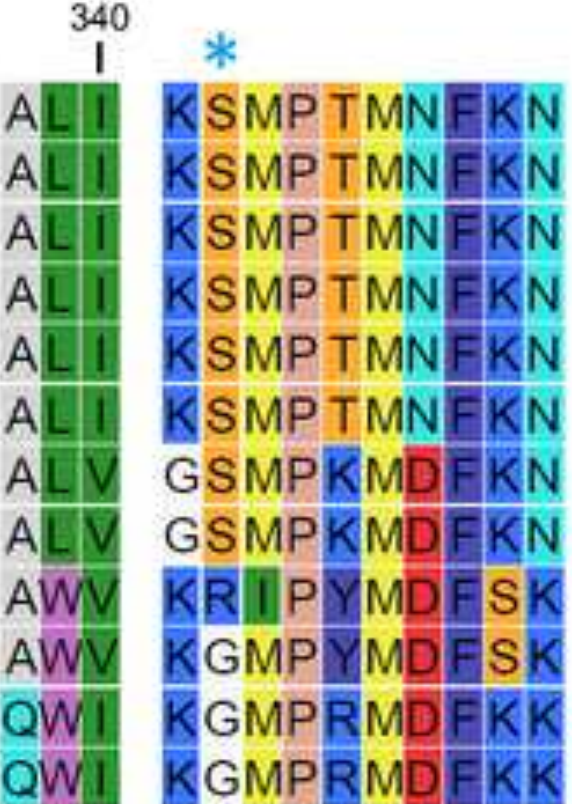

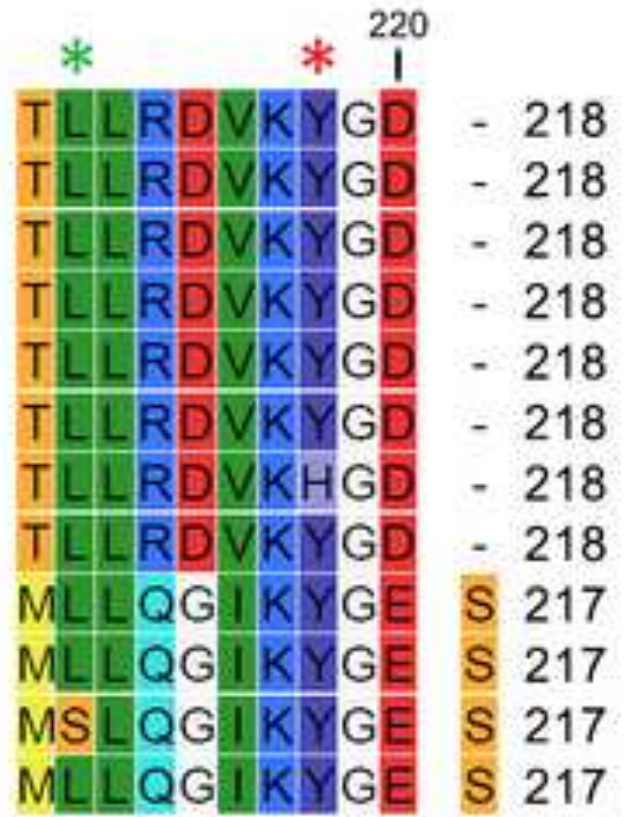


other proteins contained at least one amino acid substitution, including NS4, which was truncated due to a premature stop codon. Nine non-synonymous substitutions were observed in serotype 4 and eight in serotype 6 (Fig. 2c and d). No amino acid substitutions were observed on VP3, VP5, NS2, VP7 or NS3 of serotype 4, while VP3, VP4, NS1, VP5 and VP7 were free of amino acid substitutions in serotype 6 (Fig. 2c and d).

The AHSV-1 and AHSV-2 viruses showed amino acid substitutions in proteins VP2, VP3, VP5 and NS3 between the different plaque sizes. Furthermore, variation analysis showed that the substitutions seen in VP2, VP3 and VP5 were unique to the large plaque viruses, whereas the substitution seen in NS3 existed in the small plaque viruses as a minor sub-population (5.302\% and $5.3 \%$ respectively). Though the amino acid substitution in NS3 did not occur in all four AHSV serotypes, it should be noted that once again the substitutions in AHSV-1, -2 and -6 were all located in the C-terminal of the protein and that the types of substitutions were similar in that non-polar amino acids were replaced with polar amino acids in the high passage strain (Fig. 3a).

A single amino acid substitution, located between amino acids 340 and 360 of VP2 was observed in all four serotypes (Fig. 3b). In all four serotypes, these substitutions resulted in the replacement of either a hydrophobic residue ( $\mathrm{M}$ in AHSV-1 3 S and T in AHSV-6 4S), a polar uncharged residue (N in AHSV$23 S$ ) or a neutral G in AHSV-4 1S with a positively changed residue, K or R. Each NS3 also had one amino acid substitution in the large plaque variants of each isolate at the conserved $C$-terminal of the protein (Fig. 3a). As with VP2, a hydrophobic residue (M in AHSV-1 3S and Y in AHSV-2 3S) was replaced with a positively charged residue in the large plaque strains ( $\mathrm{K}$ and $\mathrm{H}$ respectively). In contrast, VP3 and VP5 had substitutions in the large plaque strains of both isolates, they are not located in the same regions of the proteins nor were any observed in serotype 4 or 6 .

\section{Hydropathic and antigenic properties of VP2}

VP2 is the serotype specific antigenic protein in orbiviruses, as well as being essential for virus entry into the host cell $[9,10]$. The predicted influence of each amino acid substitutions on the antigenicity of the protein was determined in relation to its location on the surface of the protein.

The amino acid substitutions were located in an area that was calculated to have a negative antigenicity value [56]. The effect of the change in amino acid in the large plaque strains of each isolate was to increase the predicted antigenicity of that region when compared to the small plaque strains (Fig. 4). Compared to their respective parent isolates, the increase in antigenicity value of that region of VP2 was on average 0.030 (48\%) in AHSV-1 LP(A), 0.065 (344\%) in AHSV-2 LP, 0.033 (205\%-fold) in Vac4 and $0.01(18 \%)$ in Vac6. Despite these predicted increases in the antigenicity of this region of VP2, the average antigenicity values remained negative.

The hydrophobicity of each protein was determined, to estimate the change that the amino acid substitutions were to have on the surface of VP2. The amino acid substitution for VP2 was predicted to occur in an area with a negative hydropathic index, and the effect of the substitution in the large plaque strains reduced the hydropathic index further (Fig. 5). The change in predicted hydrophobicity is small 
Fig. 4 Prediction of the antigenic properties of the VP2 proteins of the small and large plaque strains of each of the four AHSV isolates. In each case, the amino acid substitution in the large plaque strain causes an increase in the predicted antigenicity value in that region of the protein, though the average antigenicity value remained negative
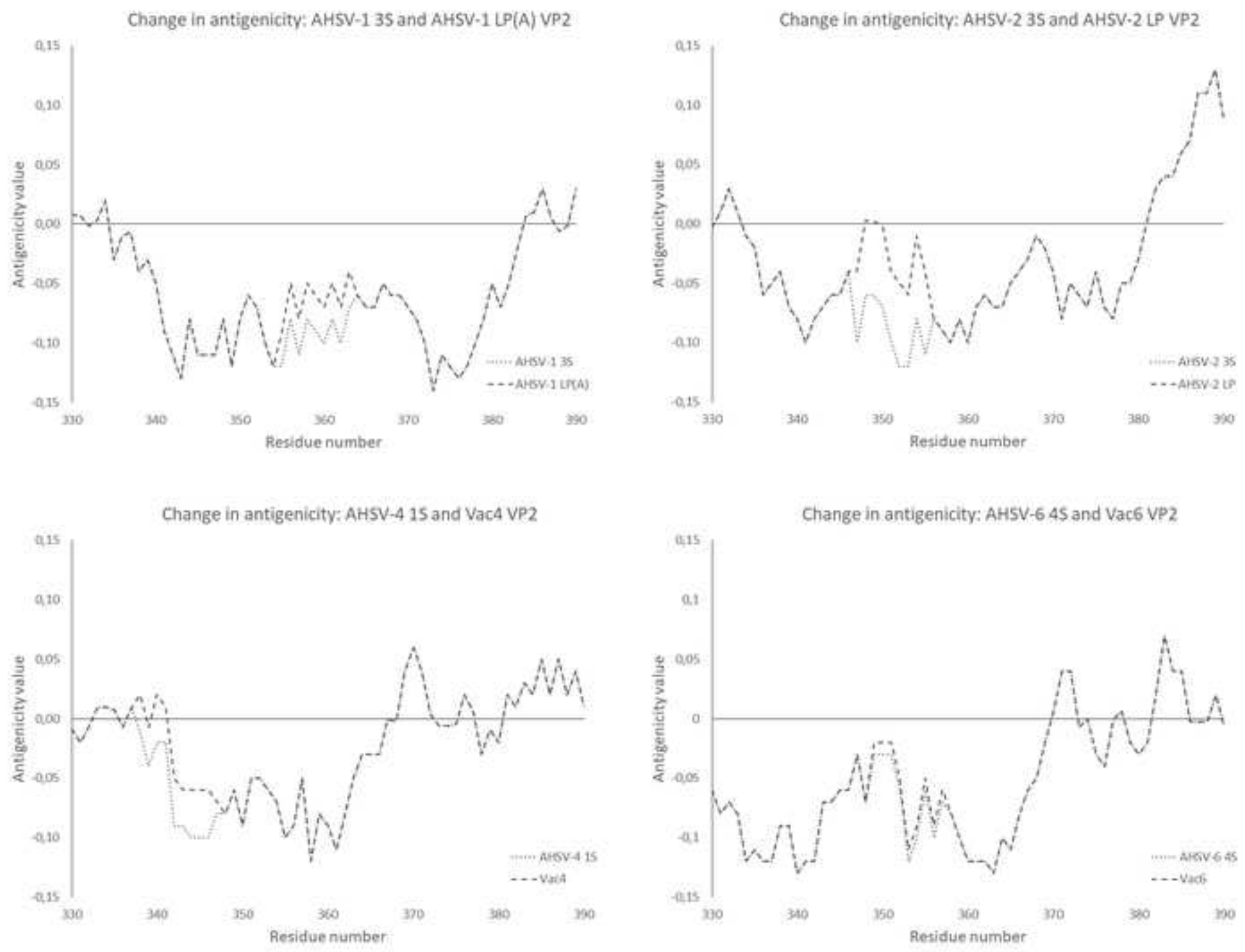
Fig. 5 Prediction of the hydropathic properties of VP2 region from 330 to 390 amino acid. This is the region where the amino acid substitution between small and large plaque strains of each isolate occurred
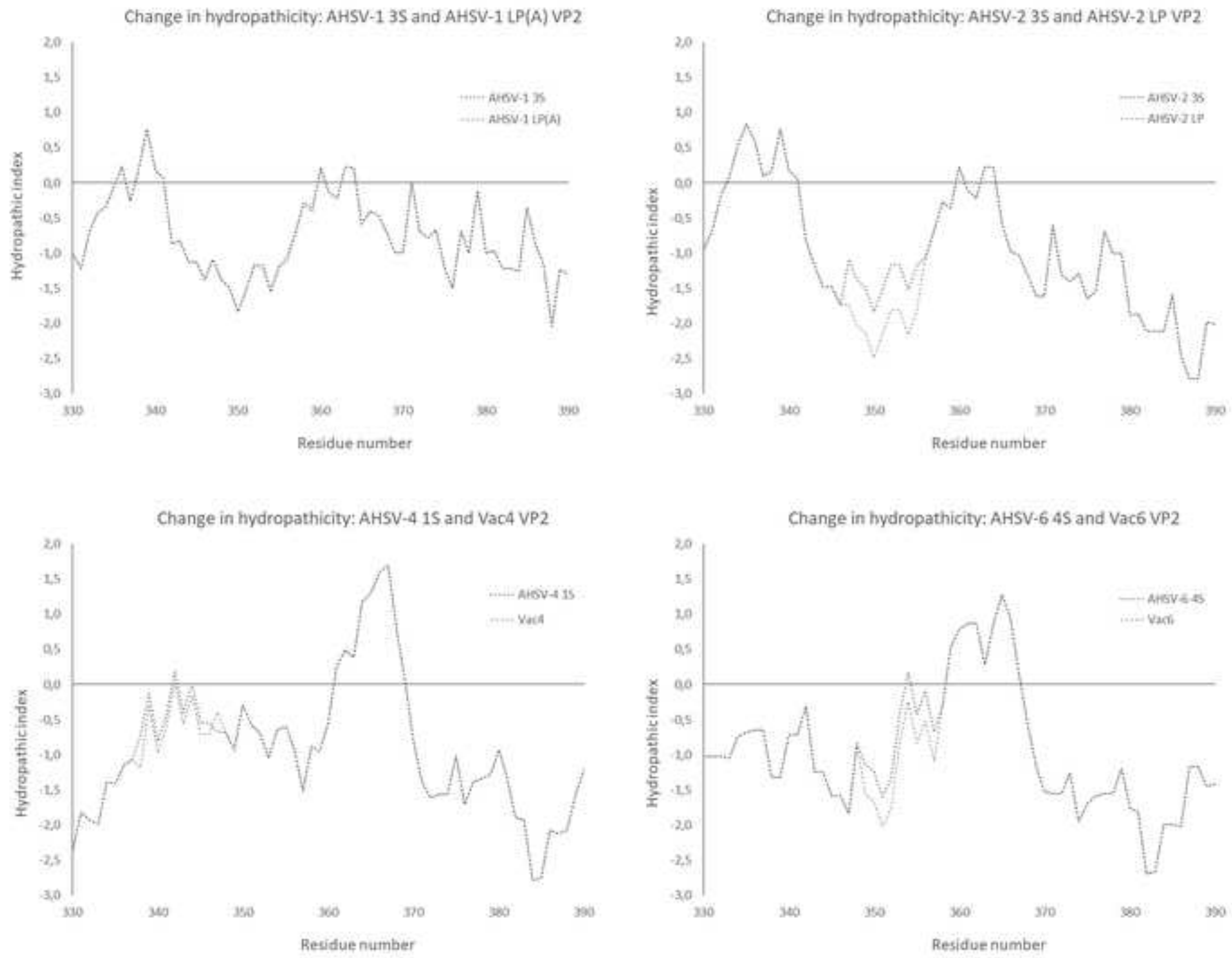
when comparing AHSV-1 $3 S$ and $L P(A)$ where the average hydropathic index is 0.044 points (5\%) lower in AHSV-1 LP(A). The change in hydropathicity is larger for the other isolates, where the index is on average 0.644 points lower in AHSV-2 LP (47\% lower), 0.2 lower in Vac4 (37\% lower), and 0.422 lower in Vac6 (56\%).

\section{Discussion}

The aim of this work was to determine if any consistent genetic variations could be observed between the genomes of related AHSV strains, expressing varying phenotypic characters in plaque morphology. As plaque size has previously been correlated to virulence $[42,43]$, it is conceivable that the genetic differences involved in plaque phenotype could be linked to the attenuation of the virus. Once identified, the effect of the genetic variations could be verified and incorporated into synthetic vaccine viruses using a reverse genetics system [57-59].

The genomes of eight AHSV strains, six of AHSV-1 and two of AHSV-2 were sequenced, and their respective plaque phenotypes determined. The six strains of AHSV-1 were categorised into small, intermediate and large plaque phenotypes. The nucleotide substitutions between these strains could be used to determine which proteins were most likely to be involved in the manifestation of the large plaque phenotype. Changes unique to the intermediate and large plaque phenotypes could not be responsible for the large plaque phenotype. Based on complete genome analysis VP4 and NS2 were omitted as possible contributors to the prescribed phenotype. Additionally, segment 9 was altered in only one of the intermediate plaque strains, and thus VP6 and NS4 could both be eliminated as causes of the large plaque phenotype in this isolate. Substitutions that were unique to the large plaque phenotype occurred in VP2, VP3 VP5 and NS3 and were selected as putative candidates to be involved in the manifestation of the large plaque phenotype. The same proteins had amino acid substitution between the strains of AHSV-2, while only VP2 contained non-synonymous SNPs when four serotypes were investigated. A subpopulation of the small plaque strains contain the same substitution in segment 10 as what is observed in the large plaque strains. As there is no corresponding large plaque morphology within these strains, it is unlikely that the NS3 protein is responsible for the shift in plaque phenotype. Similarly, the substitutions reported in AHSV-1 and AHSV-2 were not present between the variants of AHSV-4 and AHSV-6, and were thus not required for the formation of large plaques.

It was thus determined that VP2 was the only protein that was uniformly mutated between small and large plaque expressing strains of all four AHSV serotypes used in this study. The amino acid substitutions which occurred between the strains of AHSV-4 and - 6 were located in the same region as those found in AHSV-1 and -2. In each case an uncharged amino acid was replaced by a positively charged amino acid in the high passage strain of each isolate. The similarity of the changes observed, suggests that in all four serotypes the shift in plaque size is caused by the same mechanism.

Computational predictions indicated that the affected region had no influence on the antigenicity of the protein regardless of the amino acid substitution, which correlates well with a recent study on the B-cell epitopes [60]. The region in which the VP2 amino acid substitutions occurred were predicted to be 
hydrophilic in nature, and is thus likely to appear on the surface of VP2 [55]. This hydrophilic area was conserved in VP2 of each of the four serotypes used in this study, and in each serotype, the effect of the substitution was to increase the hydrophilicity of that region. Since no function has been assigned to this region of VP2, it is difficult to speculate what the effect of this modification on the protein is.

The prediction that the mutation of AHSV VP2 causes the manifestation of the large plaque phenotype agrees with a previous study on bluetongue virus (BTV), where the insertion of a large plaque segment 2 into a small plaque virus resulted in a shift from small plaques to large plaques phenotype [61]. This study also found that while the mutation of VP2 greatly reduced the virulence of BTV, it alone was not sufficient for complete attenuation [61]. A second protein mutation that greatly contributed to a reduction in virulence was NS3, though this change alone also did not result in the complete attenuation of BTV[61]. Complete attenuation was achieved through the mutation of VP2 in combination with the mutation of either VP1, VP5 or NS3 [61].

While all the tested vaccine viruses showed a mutation in VP2, it did not consistently reveal mutation in other proteins. This can be seen in the fact that the NS3 protein of the large plaque variants of three of the isolates have mutations in the C-terminal region (in each case a non-polar amino acid is replaced with a polar one), with Vac4 being the only large plaque virus in which the NS3 protein remained unchanged. The AHSV serotype 1 vaccine (Vac1) is derived from AHSV isolate 29/62 (AHSV-1), and the amino acid substitution seen in the large plaque strain NS3 proteins also occur in the vaccine virus [45].

In conclusion, the results showed that in the case of AHSV, there were consistent non-synonymous variations in the gene that codes for VP2 between large and small plaque phenotype strains of four isolates, while amino acid substitutions were reported in the C-terminus of the NS3 proteins of three of the AHSV serotypes. The protein changes reported here could have implications for the design of synthetic attenuated viruses once their individual effects have been verified in an animal model.

\section{Statement of author contributions}

MLSW: Study design and execution, preparation of manuscript

AvS: Genome sequencing and assembly, experimental set up, and reviewed the manuscript JJOK: Conceptualised the study, preparation of the manuscript

\section{Acknowledgements}

Dr Jeanni Fehrsen for critical review of the manuscript. 
Fig. 1: Plaque diameters of the AHSV-1 and AHSV-2 virus strains. Average plaque size for each strain has been indicated with a stippled line.

Fig. 2: Amino acid differences between the strains of (a) AHSV-1, (b) AHSV-2, (c) AHSV-4 and (d) AHSV-6. The approximate location of each amino acid substitution has been indicated by a black bar, and the type of change annotated.

Fig. 3: Amino acid alignments showing substitutions between the strains of AHSV-1 and AHSV-2 in (a) VP2 and (b) NS3. The location of the amino acid substitutions between strains of each isolate is indicated with either a black (AHSV-1), red (AHSV-2), blue (AHSV-4) or green (AHSV-6) asterisk.

Fig. 4: Prediction of the antigenic properties of the VP2 proteins of the small and large plaque strains of each of the four AHSV isolates. In each case, the amino acid substitution in the large plaque strain causes an increase in the predicted antigenicity value in that region of the protein, thought the average antigenicity value remained negative.

Fig. 5: Prediction of the hydropathic properties of VP2 region from 330 to 390 amino acid. This is the region where the amino acid substitution between small and large plaque strains of each isolate occurred.

Table 1: List of viruses propagated in this study.

Table 2: Average plaque size of the AHSV-1 and AHSV-2 strains.

Disclosure of potential conflicts of interest

The authors declare that they have no conflicts of interest

Involvement of human participants or animals

No human or animal subjects were used in this study

Informed consent

No human subjects were used in this study

\section{References}

1. C. H. Calisher and P. P. Mertens, Arch. Virol. Suppl. 14, 3 (1998).

2. H. J. Els and D. W. Verwoerd, Virology 38, 213 (1969).

3. R. A. Oellermann, H. J. Els, and B. J. Erasmus, Arch. Gesamte Virusforsch. 29, 163 (1970).

4. C. W. Bremer, Onderstepoort J. Vet. Res. 43, 193 (1976). 
5. C. W. Bremer, H. Huismans, and A. A. Van Dijk, J. Gen. Virol. 71, 793 (1990).

6. M. J. Grubman and S. A. Lewis, Virology 186, 444 (1992).

7. M. Belhouchet, F. M. Jaafar, A. E. Firth, J. M. Grimes, P. P. C. Mertens, and H. Attoui, PLoS One 6, e25697 (2011).

8. L. Zwart, C. A. Potgieter, S. J. Clift, and V. van Staden, PLoS One e0124281 (2015).

9. H. Huismans and B. J. Erasmus, Onderstepoort J. Vet. Res. 48, 51 (1981).

10. S. S. Hassan and P. Roy, J. Virol. 73, 9832 (1999).

11. M. Du Plessis and L. H. Nel, Virus Res. 47, 41 (1997).

12. J. L. Martínez-Torrecuadrada, J. P. M. Langeveld, A. Venteo, A. Sanz, K. Dalsgaard, W. D. O. Hamilton, R. H. Meloen, and J. I. Casal, Virology 257, 449 (1999).

13. L. Stassen, H. Huismans, and J. Theron, Arch. Virol. 156, 711 (2011).

14. V. Manole, P. Laurinmaki, W. Van Wyngaardt, C. A. Potgieter, I. M. Wright, G. J. Venter, A. A. Van Dijk, B. T. Sewell, and S. J. Butcher, J. Virol. 86, 7858 (2012).

15. P. Roy, A. Fukusho, G. D. Ritter, and D. Lyon, Nucleic Acids Res. 16, 11759 (1988).

16. F. T. Vreede and H. Huismans, Arch. Virol. 143, 413 (1998).

17. T. Urakawa, D. G. Ritter, and P. Roy, Nucleic Acids Res. 17, 7395 (1989).

18. M. Boyce, J. Wehrfritz, R. Noad, and P. Roy, J. Virol. 78, 3994 (2004).

19. H. Le Blois, T. French, P. P. C. Mertens, J. N. Burroughs, and P. Roy, Virology 189, 757 (1992).

20. N. Ramadevi, N. J. Burroughs, P. P. C. Mertens, I. M. Jones, and P. Roy, Proc. Natl. Acad. Sci. U. S. A. 95, 13537 (1998).

21. M. E. Stewart and P. Roy, FEBS Open Bio 5, 138 (2015).

22. P. Roy, A. Adachi, T. Urakawa, T. F. Booth, and C. P. Thomas, J. Virol. 64, 1 (1990).

23. P. J. De Waal and H. Huismans, Arch. Virol. 150, 2037 (2005).

24. N. Stäuber, J. Martinez-Costas, G. Sutton, K. Monastyrskaya, and P. Roy, J. Virol. 71, 7220 (1997).

25. E. Matsuo and P. Roy, J. Virol. 83, 8842 (2009).

26. R. J. Owens, C. Limn, and P. Roy, J. Virol. 78, 6649 (2004).

27. M. Boyce, C. C. P. Celma, and P. Roy, Virol. J. 9, 178 (2012).

28. C. P. Thomas, T. F. Booth, and P. Roy, J. Gen. Virol. 71, 2073 (1990). 
29. A. D. Hyatt, A. R. Gould, B. Coupar, and B. T. Eaton, J. Gen. Virol. 72, 2263 (1991).

30. M. A. Stoltz, C. F. van der Merwe, J. Coetzee, and H. Huismans, Onderstepoort J. Vet. Res. 63, 57 (1996).

31. T. L. Meiring and H. Huismans, Arch. Virol. 154, 263 (2009).

32. M. Ratinier, A. E. Shaw, G. Barry, Q. Gu, L. Di Gialleonardo, A. Janowicz, M. Varela, R. E. Randall, M. Caporale, and M. Palmarini, J. Virol. 90, 5427 (2016).

33. N. D. Diouf, E. Etter, M. M. Lo, M. Lo, and A. J. Akakpo, Vet. Rec. 172, 152 (2013).

34. R. M. Du Toit, Onderstepoort J. Vet. Res. 19, 7 (1944).

35. N. K. Blackburn, L. Searle, and R. J. Phelps, J. Entomol. Soc. South. Afr. 48, 331 (1985).

36. P. S. Mellor, J. Boned, C. Hamblin, and S. Graham, Epidemiol. Infect. 105, 447 (1990).

37. R. Meiswinkel and J. T. Paweska, Prev. Vet. Med. 60, 243 (2003).

38. P. G. Howell, in Emerg. Dis. Anim. (FAO Agricultural Studies, Rome, 1963), pp. 71-108.

39. V. S. Binepal, B. N. Wariru, F. G. Davies, R. Soi, and R. Olubayo, Vet. Microbiol. 31, 19 (1992).

40. F. Fasina, A. C. Potgieter, A. Ibironke, B. Bako, D. Bwala, and P. Kumbish, J. Equine Vet. Sci. 28, 167 (2008).

41. N. J. MacLachlan and A. J. Guthrie, Vet. Res. 41, 35 (2010).

42. B. J. Erasmus, in Proc. 4th Int. Conf. Equine Infetious Dis., edited by J. T. Bryans and H. Gerber (Veterinary Publications International, Princetown, 1978), pp. 401-403.

43. B. J. Erasmus, in Proc. 3rd Int. Conf. Equine Infect. Dis. (Paris, 1972), pp. 1-11.

44. A. J. Guthrie, P. Coetzee, D. P. Martin, C. W. Lourens, E. H. Venter, C. T. Weyer, C. Joone, M. Le Grange, C. K. Harper, P. G. Howell, and N. J. MacLachlan, Genome Announc. 3, e01375 (2015).

45. A. J. Guthrie, P. Coetzee, D. P. Martin, C. W. Lourens, E. H. Venter, C. T. Weyer, C. Joone, M. Le Grange, C. K. Harper, P. G. Howell, and N. J. MacLachlan, Genome Announc. 3, e00814 (2015).

46. A. C. Potgieter, I. M. Wright, and A. A. Van Dijk, Genome Announc. 3, e00921 (2015).

47. I. Macpherson and M. Stoker, Virology 16, 147 (1962).

48. M. Sato, H. Tanaka, T. Yamada, and N. Yamamoto, Arch. Virol. 54, 333 (1977).

49. R. A. Oellermann, Onderstepoort J. Vet. Res. 37, 137 (1970).

50. M. D. Abràmoff, P. J. Magalhães, and S. J. Ram, Biophotonics Int. 11, 36 (2004).

51. H. B. Mann and D. R. Whitney, Ann. Math. Stat. 18, 50 (1947)

52. A. C. Potgieter, N. A. Page, J. Liebenberg, I. M. Wright, O. Landt, and A. A. van Dijk, J. Gen. Virol. 
90, 1423 (2009).

53. K. Clark, I. Karsch-Mizrachi, D. J. Lipman, J. Ostell, and E. W. Sayers, Nucleic Acids Res. 44, D67 (2016).

54. P. Artimo, M. Jonnalagedda, K. Arnold, D. Baratin, G. Csardi, E. De Castro, S. Duvaud, V. Flegel, A. Fortier, E. Gasteiger, A. Grosdidier, C. Hernandez, V. Ioannidis, D. Kuznetsov, R. Liechti, S. Moretti, K. Mostaguir, N. Redaschi, G. Rossier, I. Xenarios, and H. Stockinger, Nucleic Acids Res. 40, W597 (2012).

55. J. Kyte and R. F. Doolittle, J. Mol. Biol. 157, 105 (1982).

56. G. W. Welling, W. J. Weijer, R. van der Zee, and S. Welling-Wester, FEBS Lett. 188, 215 (1985).

57. E. Matsuo, C. C. P. Celma, and P. Roy, FEBS Lett. 584, 3386 (2010).

58. Y. Kaname, C. C. P. Celma, Y. Kanai, and P. Roy, J. Gen. Virol. 94, 2259 (2013).

59. A. M. Conradie, L. Stassen, H. Huismans, C. A. Potgieter, and J. Theron, Virology 499, 144 (2016).

60. E. M. Mathebula, F. E. Faber, W. van Wyngaardt, A. van Schalkwyk, A. Pretorius, and J. Fehrsen, Onderstepoort J. Vet. Res. 84, a1313 (2017).

61. A. Janowicz, M. Caporale, A. Shaw, S. Gulletta, L. Di Gialleonardo, M. Ratinier, and M. Palmarini, J. Virol. 89, 5238 (2015). 